\title{
Forward Model of Electrical Impedance GeoTomography with Point Electrode Model Based on Mixed Finite Element Method: MATLAB Programming Notes
}

\author{
A.D. Garnadi ${ }^{1}$ \& C. Bahriawati ${ }^{1, \dagger}$
}

31 December 2017

\author{
${ }^{1}$ Dept. Mathematics, Institut Pertanian Bogor, \\ Jl. Meranti, Kampus IPB Darmaga, Bogor, 16680 Indonesia \\ $\dagger$ Deceased.
}

\begin{abstract}
We address the need of forward model of electrical impedance geotomography using Mixed Finite Element Method. We use the mixed finite-element method with the standard P1- P0 pair for triangular decompositions for calculating model responses, which makes it possible to compute both the electric potential and the electric field vector economically.

For MATLAB implementation, the routine available is easy to modify without hasle.

Electrical impedance geotomography (GeoEIT) is a low-cost non-invasive imaging modality. It has the potential to be of great value in clinical diagnosis. One of the major problems in GeoEIT with complex geometry shape is its high demand in computation capability, power, and memory. A mixed finite element method (MFEM) is proposed to calculate the forward problem accurately. Compared with the traditional FEM, a smaller number of nodes and elements with the proposed method are required to achieve the same accuracy in our numerical computation model. The results demonstrate the efficiency of the MFEM in GeoEIT simulation. In the forward solution, it is capable of achieving better accuracy using less computational time and memory with MFEM.
\end{abstract}

\section{Electrical Impedance GeoTomography as parameter identification problem}

In the study of a direct electrical current in a bounded object $\Omega$, the governing equation is the following elliptic partial differential equation:

$$
-\nabla(\sigma \nabla u)=q \quad \text { in } \quad \Omega
$$


where $\Omega \in R^{d},\{d=2,3\}$ is a bounded domain, $\sigma: \Omega \rightarrow R$ denotes the conductivity coefficient, $u: \Omega \rightarrow R$ is electrical potential, $q:=I\left(\delta\left(r-s_{i}\right)-\delta\left(r-s_{j}\right)\right)$ is current injection pair through point electrode at $s_{i, j}, i \neq j, 1 \leq i, j, \leq N$, where $N$ denoting the number of electrodes. When the current source term $q$ and the coefficient $\sigma$ is given, with an appropriate prescribed boundary information for solution $u$ so that the direct (forward) problem of $u$ is uniquely solvable in $\Omega$. Conversely, the recovery information about the coefficient of conductivity $\sigma$, given the pair $(q, g)$ is given, where $g\left(r_{k}\right):=u\left(r_{k}\right)$ is measured potential at point electrodes at $r_{k}$ due to current injection pattern $q$, is an inverse problem with $\sigma$ being sensitive due to perturbation in $u$ and $q$. The recovery of $\sigma$ from point electrodes measurements $u$ and $q$, utilizing the equation (1), that is called Electrical Impedance GeoTomography with point electrode models.

\section{Governing Equation}

Let us restrict the whole space to conductive half space $\Omega$, where $\Omega$ is the bounded and open subset of $R^{2}$ having a piecewise continuous boundary $\Gamma(=\partial \Omega)$. denotes the ground surface $(\mathrm{z}=0)$, and $\Gamma_{2}=\partial \Omega \backslash \Gamma_{1}$, write $\rho=1 / \sigma$. The governing first-order system for electric resistivity problem in the domain $\Omega$ is expressed by the following Poisson equation with mixed boundary condition ([2]).

$$
\begin{aligned}
\nabla u(x, z) & =-\rho(x, z) \mathbf{E}(x, z) \text { in } \Omega \\
\nabla . \mathbf{E}(x, z) & =\frac{I}{\Delta S}\left[\left(\delta\left(x-x_{s}^{+}\right) \delta\left(z-z_{s}^{+}\right)\right)-\left(\delta\left(x-x_{s}^{-}\right) \delta\left(z-z_{s}^{-}\right)\right)\right] \text {in } \Omega \\
\mathbf{n . E}(x, z) & =0 \text { on } \Gamma_{1}, \\
u(x, z) & =0 \text { on } \Gamma_{2}
\end{aligned}
$$

where $I$ is the current in ampere, $\left(x_{s}^{+}, z_{s}^{+}\right)$and $\left(x_{s}^{-}, z_{s}^{-}\right)$is the coordinate of point sources pair generated by an injected charge, $\Delta S$ is the elemental area about the chargeinjected point and $\mathbf{n}$ is the outer normal vector at the boundary of $\Omega$.

\section{Mixed finite element method ([1],[3])}

Given a triangulation $\mathcal{T}_{h}$ by simplexes $T$ of a domain $\bar{\Omega}$, we introduce a finite-dimensional subspace $W_{h}$ of $\left(L^{2}(\Omega)\right)^{2}$ and a finite-dimensional subspace $M_{h}$ of $H^{1}(\Omega)$ expressed as

$$
\begin{aligned}
& W_{h}=\left\{q \in W:\left.\forall T \in T q\right|_{T} \in\left(P_{0}(T)\right)^{2}\right\}, \\
& M_{h}=\left\{v \in M: \forall T \in T_{h} ;\left.v\right|_{T} \in P_{1}(T)\right\}
\end{aligned}
$$

Therefore Galerkin finite-element method is defined as

$$
\int_{\Omega} \rho(x, z) \mathbf{E}_{h}(x, z) \bullet \mathbf{p}(x, z) d x d z+\int_{\Omega} \mathbf{p}(x, z) \bullet \nabla u_{h}(x, z) d x d z=0 \quad \text { for all } \quad \mathbf{p} \in
$$




$$
\begin{array}{r}
\int_{\Omega} \mathbf{E}_{h}(x, z) \bullet \nabla v_{h}(x, z) d x d z= \\
\int_{\Omega} \frac{I}{\Delta S}\left[\left(\delta\left(x-x_{s}^{+}\right) \delta\left(z-z_{s}^{+}\right)\right)-\left(\delta\left(x-x_{s}^{-}\right) \delta\left(z-z_{s}^{-}\right)\right)\right] v_{h} d x d z \text { for all } v_{h} \quad \in \quad M_{h}
\end{array}
$$

and we find $\left(\mathbf{E}_{h}, u_{h}\right) \in \mathbf{W}_{h} \times M_{h}$ satisfying equations (8) and (9). In equation (8,9), we divide the region by triangular element and define the potential and electric field using basis functions of finite dimensional space $\mathbf{W}_{h}$ and $M_{h}$ as

$$
\begin{aligned}
\mathbf{E}_{h}(x, z) & =\sum_{i=1}^{2 M} e_{i} \Psi_{i}, \\
u_{h}(x, z) & =\sum_{i=1}^{N} u_{i} \phi_{i}(x, z),
\end{aligned}
$$

where $N$ is the number of nodal points of domain, $M$ is the number of triangular elements, and $\Psi_{i}(x, z)$ and $\phi_{i}(x, z)$ are the basis functions of $\mathbf{W}_{h}$ and $M_{h}$, respectively. Then, equations $(8,9)$ can be written in a matrix form as

$$
\mathbf{K} \mathbf{U}=\mathbf{F}
$$

with

$$
\begin{aligned}
& \mathbf{K}=\left[\begin{array}{ll}
\mathbf{B} & \mathbf{C} \text {; }
\end{array}\right. \\
& \left.\mathbf{C}^{T} \mathbf{O}\right] \\
& \mathbf{U}=\left[\begin{array}{llllll}
e_{1} & \ldots & e_{2 M} & u_{1} & \ldots & u_{N}
\end{array}\right]^{T} \\
& \mathbf{F}=\left[\begin{array}{ll}
0 & -\mathbf{f}
\end{array}\right]^{T}
\end{aligned}
$$

From equation (5), we can obtain solution vectors $\mathbf{u}_{h}=\left(u_{1}, \ldots, u_{N}\right)$ and $\mathbf{e}_{h}=\left(e_{1}, \ldots e_{2 M}\right)$ using

$$
\begin{aligned}
\mathbf{C}^{T} \mathbf{B}^{-1} \mathbf{C} \mathbf{u}_{h} & =\mathbf{f}, \\
\mathbf{e}_{h} & =-\mathbf{A}^{-1} \mathbf{C u}_{h}
\end{aligned}
$$

Since $\Psi_{i}$ is constant, $\mathbf{A}$ is a diagonal matrix. Therefore, computing time required for obtaining $\mathbf{u}_{h}$ and $\mathbf{e}_{h}$ is compatible with that required when we use other numerical methods (finite element or finite difference method).

Programming Note:

The MATLAB implementation in [1] is almost straightforward to implement, except the need to modify the following terms :

$$
\int_{\Omega} \rho(x, z) \mathbf{E}_{h}(x, z) \bullet \mathbf{p}(x, z) d x d z .
$$

Assuming that $\rho(x, z)$ is piece-wise continuous, and if we choose Triangulation of the domain such that $\left.\rho(x, z)\right|_{T_{i}}=\rho_{i}, i=1, \cdots, N e$, where $\mathcal{T}=\left\{T_{i} \subset \Omega ; T_{1}, \cdots, T_{N e}\right\}$, is the triangulation of $\Omega$. 


\section{Example}

In order to examine the implementation of above setting, we use it for generating synthetic data, we take a simple 2 dimensional case, as shown in Figure 1.

A pair of electrodes, situated at nodes 7 and 8 , and resistivity set at $1 \mathrm{Ohm}$, in the domain except at triangle 3 which have value $2 \mathrm{Ohm}$.
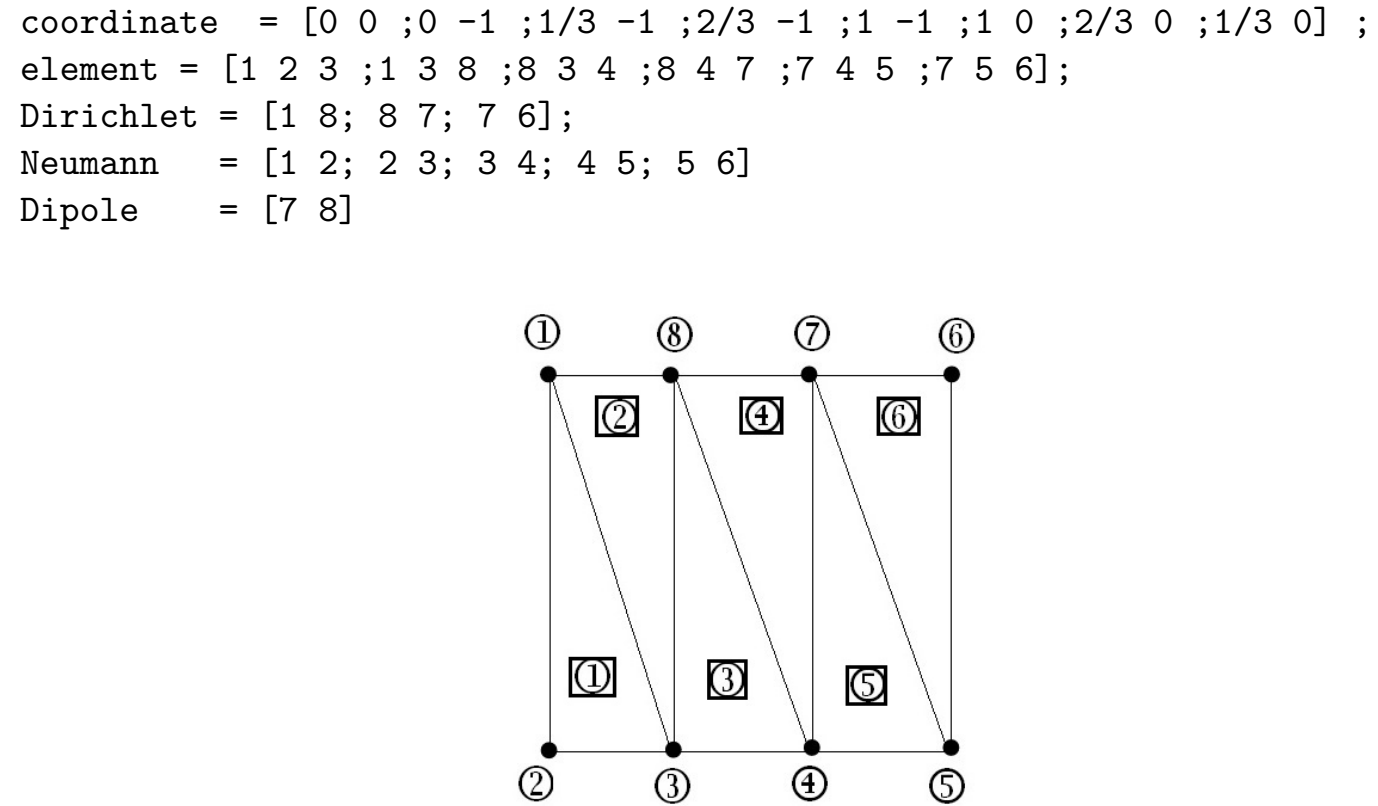

Figure 1: Mesh consists of six elements in $\Omega$

\section{Conclusions}

Unlike in the conventional DC modeling, we are enforced to use the mixed finite-element method with $P_{1}-P_{0}$ triangular element which guarantees to compute electric field and potential simultaneously. Off the shelf MATLAB library for Mixed Finite Element Method is straightforward to solve the Governing equations.

\section{Acknowledgement}

This work partially funded by 083/SP2H/PL/Dit.Litabmas/II/2015.

\section{References}

[1] Bahriawati, C., and C. Carstensen. "Three MATLAB implementations of the lowestorder Raviart-Thomas MFEM with a posteriori error control." Computational 
Methods in Applied Mathematics Comput. Methods Appl. Math. 5, no. 4 (2005): 333-361.

[2] Dey, A., and Morrison, H. F., 1979, Resistivity modeling for arbitrarily shaped two-dimensional structures: Geophys. Prosp., 27, 106-136.

[3] Ha, Taeyoung and Pyun, Sukjoon and Shin, Changsoo, 2006, Efficient electric resistivity inversion using adjoint state of mixed finite-element method for Poissons equation, Journal of Computational Physics, v 214,1,pp 171-186, 
${ }_{103}$ MATLAB Scripts

104 The script for case 1 will be put in here 